\title{
Impact of Pilot Assisted Channel State Estimation on Multiple Antenna Multiuser TDD Systems with Spatial Filtering
}

\author{
Dragan Samardzija \\ Bell Laboratories, Lucent Technologies \\ 791 Holdel-Keyport Road \\ Holmdel, NJ 07733, USA \\ Email: dragan@bell-labs.com
}

\author{
Liang Xiao \\ WINLAB, Rutgers University \\ 671 Route 1 South \\ North Brunswick, NJ 08902, USA \\ Email: 1xiao@winlab.rutgers.edu
}

\author{
Narayan Mandayam \\ WINLAB, Rutgers University \\ 671 Route 1 South \\ North Brunswick, NJ 08902, USA \\ Email: narayan@winlab.rutgers.edu
}

\begin{abstract}
In this paper we study a multiple antenna multiuser TDD system that uses pilot assisted channel state estimation. Specifically, we consider a wireless system with multiple antennas at the base station and a number of mobile terminals each with a single antenna. We analyze the performance of the uplink multiuser detection and downlink transmitter optimization that are based on linear spatial filtering. Using a block fading channel model we analyze how a lower bound on the achievable rates depends on the power allocated to the pilots as well as the background noise level. Effects of the coherence time and temporal correlations in wireless channels are also analyzed.
\end{abstract}

\section{INTRODUCTION}

In multiuser wireless systems application of multiple antennas appears to be one of the most promising solutions leading to even higher data rates and/or the ability to support greater number of users. Multiuser detection schemes that exploit multiple antennas provide significant increase of the uplink data rates [1]. Likewise, multiple antenna transmitter optimization schemes provide significant increase of the downlink data rates [2]-[4]. Spatial filtering at the uplink receiver and spatial prefiltering at the downlink transmitter are examples of linear multiuser detection and transmitter optimization, respectively. In the literature, these specific solutions are also known as receiver and transmitter beamforming.

Both the multiuser detection and transmitter optimization rely heavily on the availability of the channel state information (CSI) at the receiver and transmitter, respectively. Impact of delayed CSI on the downlink data rates is reported in [5]. In systems where the uplink and downlink channel states are mutually independent a CSI feedback is needed to support downlink transmitter optimization. Wireless systems that apply frequency division duplexing (FDD) typically have mutually independent downlink and uplink channel states. For example, a corresponding CSI feedback scheme is proposed in [6].

Unlike the FDD systems, systems with the time division duplexing (TDD) have very high correlation between successive uplink and downlink channel states. Due to the reciprocity of the uplink and downlink channels, in an ideal case of static environment, the channel states are identical. The above property supports application of the downlink transmitter optimization without an explicit CSI feedback. Specifically, during the uplink transmission interval, the uplink receiver estimates the uplink channel state. Then, during the following downlink transmission interval, the transmitter can apply the channel state estimate to optimize the downlink transmission.
In this study we consider a multiple antenna multiuser TDD system that uses pilot assisted channel state estimation. Specifically, we consider a wireless system with multiple antennas at the base station and a number of mobile terminals each with a single antenna. We study the performance of the uplink multiuser detection and downlink transmitter optimization that are based on linear spatial filtering. Using a characterization of noise due to estimation errors, we present a lower bound on the achievable rates as a function of the power allocated to the pilots as well as the background noise level. In addition, effects of the coherence time and temporal correlations in wireless channels are considered.

The paper is organized as follows. In Section II the uplink model is introduced and the lower bound on the achievable rates is presented. Equivalent results for the downlink are given in Section III. The MMSE linear spatial filter is introduced in Section IV with the corresponding numerical results in Section $\mathrm{V}$. We conclude in Section VI.

\section{UPLINK}

The system consists of $M$ antennas at the base station and $N$ mobile terminals (each with a single antenna). The uplink signal received at the base station is presented in a vector form as

$$
\begin{aligned}
& \mathbf{y}^{u l}=\mathbf{H}^{u l} \mathbf{x}^{u l}+\mathbf{n}^{u l}, \\
& \mathbf{y}^{u l} \in \mathcal{C}^{M}, \mathbf{x}^{u l} \in \mathcal{C}^{N}, \mathbf{n}^{u l} \in \mathcal{C}^{M}, \mathbf{H}^{u l} \in \mathcal{C}^{M \times N}
\end{aligned}
$$

where $\mathbf{x}^{u l}=\left[x_{1}^{u l}, \cdots, x_{N}^{u l}\right]^{\mathrm{T}}$ with $x_{i}^{u l}(i=1, \cdots, N)$ being a signal transmitted from mobile terminal $i . \mathbf{n}^{u l}$ is AWGN $\left(\mathrm{E}\left[\mathbf{n}^{u l}\left(\mathbf{n}^{u l}\right)^{\mathrm{H}}\right]=N_{0} \mathbf{I}_{M \times M}\right) . \mathbf{H}^{u l}$ is the uplink channel matrix. Note that the channels are assumed to be constant for the duration of uplink and downlink transmission period. In other words, we assume a block fading model. Further, $h_{m n}^{u l}$ is the $m$ th row and $n$th column element of the matrix $\mathbf{H}^{u l}$ corresponding to the uplink channel state between mobile terminal $n$ and base station antenna $m$. The signal transmitted from mobile terminal $i$ is

$$
\begin{aligned}
x_{i}^{u l}(t) & =\underbrace{\sum_{k=1}^{K^{u l}-N} \sqrt{P_{d}^{u l}} d_{i k}^{u l} s_{k}^{u l}(t)}_{\text {Data }}+ \\
& +\underbrace{\sqrt{P_{p}^{u l}} s_{\left(K^{u l}-N+i\right)}^{u l}(t)}_{\text {Pilot }}, 0 \leq t \leq K^{u l} T_{\text {sym }},(2)
\end{aligned}
$$


where $s_{k}^{u l}(t)\left(k=1, \cdots, K^{u l}\right)$ are mutually orthogonal unitenergy waveforms. $d_{i k}^{u l}$ is a data symbol transmitted from mobile terminal $i$ on waveform $s_{k}^{u l}(t) . d_{i k}^{u l}$ is assumed to be a circularly symmetric complex random variable with complex Gaussian distribution $\mathcal{N}_{\mathcal{C}}(0,1)$. Note that the datacarrying waveforms $s_{k}^{u l}(t)\left(k=1, \cdots, K^{u l}-N\right)$ are used by each mobile terminal. In other words, every data-carrying signal dimension is reused $N$ times. Unlike the data-carrying waveforms, the pilot waveforms are not reused, i.e., the waveform $s_{\left(K^{u l}-N+i\right)}^{u l}(t)$ is used exclusively as a pilot for mobile terminal $i$. Each data-carrying waveform is transmitted with the power $P_{d}^{u l}$, while the pilot waveform with the power $P_{p}^{u l}$. The duration of the transmission is limited to $K^{u l}$ symbol periods, corresponding to the uplink transmission interval.

At the receiver we assume sampling with the period $T_{\text {sym }}=$ $1 / B$, where $B$ is the bandwidth of the transmitted signal in (2). Thus, the $i$ th component of the vector $\mathrm{x}^{u l}$ (i.e., $x_{i}^{u l}$ ) corresponds to a sample of the signal $x_{i}^{u l}(t)$.

In the above definition of the transmitted signal we assume that the pilots are orthogonal to the data-carrying portion of the signal, and to each other. We do not claim that this arrangement is optimal, but we believe that it is practical.

We will observe the performance of the system with respect to the amount of transmitted energy that is allocated to the pilot (percentage wise). This percentage is denoted as $\mu^{u l}$ and is given as

$$
\mu^{u l}=\frac{P_{p}^{u l}}{\left(K^{u l}-N\right) P_{d}^{u l}+P_{p}^{u l}} 100[\%],
$$

while the average transmit power is

$$
P^{u l}=\frac{\left(K^{u l}-N\right) P_{d}^{u l}+P_{p}^{u l}}{K^{u l}} .
$$

Using the pilot assisted estimation, the maximum likelihood estimate of the uplink channel matrix $\mathbf{H}^{u l}$ is

$$
\widehat{\mathbf{H}}^{u l}=\mathbf{H}^{u l}+\mathbf{H}_{e}^{u l}
$$

where $\mathbf{H}_{e}^{u l}$ is the estimation error matrix whose entries are modeled as independent identically distributed random variables with complex Gaussian distribution $\mathcal{N}_{\mathcal{C}}\left(0, N_{0} / P_{p}^{u l}\right)$.

The decision statistics used to detect the data transmitted on the uplink is

$$
\hat{\mathbf{y}}^{u l}=\mathbf{W}^{u l} \mathbf{y}^{u l}, \hat{\mathbf{y}}^{u l} \in \mathcal{C}^{N}, \mathbf{W}^{u l} \in \mathcal{C}^{N \times M},
$$

where $\mathbf{W}^{u l}$ is a spatial filter used to suppress the uplink multiuser interference. The spatial filter is a function of the channel state estimate, i.e., $\mathbf{W}^{u l}=f\left(\widehat{\mathbf{H}}^{u l}\right)$. As a common practice, the detection procedure assumes that the channel state is perfectly estimated, and that $\widehat{\mathbf{H}}^{u l}$ corresponds to the true channel state. Let us rewrite the above expression as

$$
\begin{aligned}
\hat{\mathbf{y}}^{u l} & =\mathbf{W}^{u l}\left(\mathbf{H}^{u l} \mathbf{x}^{u l}+\mathbf{n}^{u l}\right)= \\
& =\mathbf{W}^{u l} \widehat{\mathbf{H}}^{u l} \mathbf{x}^{u l}+\underbrace{\mathbf{W}^{u l}\left(\mathbf{n}^{u l}-\mathbf{H}_{e}^{u l} \mathbf{x}^{u l}\right)}_{\text {Effective noise vector }}
\end{aligned}
$$

where the second term is the effective noise vector capturing both the AWGN and noise due to imperfect knowledge of the true uplink channel state. The decision statistics corresponding to the uplink of mobile terminal $i$ is

$$
\begin{aligned}
\hat{y}_{i k}^{u l} & =\sqrt{P_{d}^{u l}} d_{i k}^{u l} \overline{\mathbf{w}}_{i}^{u l} \hat{\mathbf{h}}_{i}^{u l}+ \\
& +\underbrace{\sum_{j=1, j \neq i}^{N} \sqrt{P_{d}^{u l}} d_{j k}^{u l} \overline{\mathbf{w}}_{i}^{u l} \mathbf{h}_{j}^{u l}}_{\text {Multiple access interference }}+ \\
& +\underbrace{\overline{\mathbf{w}}_{i}^{u l} \mathbf{n}^{u l}-\sqrt{P_{d}^{u l}} d_{i k}^{u l} \overline{\mathbf{w}}_{i}^{u l} \mathbf{h}_{e i}^{u l}}_{\text {Effective noise }}
\end{aligned}
$$

where $\overline{\mathbf{w}}_{i}^{u l}$ is the $i$ th row vector of the matrix $\mathbf{W}^{u l}, \hat{\mathbf{h}}_{i}^{u l}$ is the $i$ th column vector of the matrix $\hat{\mathbf{H}}^{u l}, \mathbf{h}_{j}^{u l}$ is the $j$ th column vector of the matrix $\mathbf{H}^{u l}$ and $\mathbf{h}_{e i}^{u l}$ is the $i$ th column vector of the matrix $\mathbf{H}_{e}^{u l}$, all for $k=1, \cdots,\left(K^{u l}-N\right)$.

Based on the above a lower bound on the uplink data rate for mobile terminal $i$ is

$$
\begin{gathered}
C_{i}^{u l} \geq R_{i}^{u l}=\frac{K^{u l}-N}{K^{u l}} \mathrm{E}_{\mathbf{H}^{u l}, \hat{\mathbf{H}}^{u l}, \mathbf{H}_{e}^{u l}}\left[\log _{2}(1+\right. \\
\left.\left.+\frac{P_{d}^{u l}\left|\overline{\mathbf{w}}_{i}^{u l} \hat{\mathbf{h}}_{i}^{u l}\right|^{2}}{\sum_{j=1, j \neq i}^{N} P_{d}^{u l}\left|\overline{\mathbf{w}}_{i}^{u l} \mathbf{h}_{j}^{u l}\right|^{2}+N_{0}\left|\overline{\mathbf{w}}_{i}^{u l}\right|^{2}+P_{d}^{u l}\left|\overline{\mathbf{w}}_{i}^{u l} \mathbf{h}_{e i}^{u l}\right|^{2}}\right)\right] .
\end{gathered}
$$

The term $\left(K^{u l}-N\right) / K^{u l}$ is introduced because out $K^{u l}$ available signal dimensions, $N$ signal dimensions are used for the pilots. In the above expression, equality holds if the effective noise is AWGN with respect to the transmitted signal. If the effective noise is not AWGN, then the above rate represents the worst-case scenario, i.e., the lower bound [7], [8]. In achieving the above rate, the receiver assumes that the effective noise is independent of the transmitted data with Gaussian spatially white distribution. In addition, in the above expression $R_{i}^{u l}$ represents an achievable rate for reliable transmission (error-free) for the specific estimation procedure assumed. Knowing the channel response perfectly or using a better channel estimation scheme (e.g., a decision driven scheme) may result in higher achievable rates.

\section{DOWNLINK}

The downlink signal received at the mobile terminals is presented in a vector form as

$$
\begin{aligned}
& \mathbf{y}^{d l}=\mathbf{H}^{d l} \mathbf{W}^{d l} \mathbf{x}^{d l}+\mathbf{n}^{d l}, \\
& \mathbf{y}^{d l} \in \mathcal{C}^{N}, \mathbf{x}^{d l} \in \mathcal{C}^{N}, \mathbf{n}^{d l} \in \mathcal{C}^{N}, \mathbf{H}^{d l} \in \mathcal{C}^{N \times M}, \mathbf{W}^{d l} \in \mathcal{C}^{M \times N}
\end{aligned}
$$

where the $i$ th component of the vector $\mathbf{y}^{d l}$ is the signal received at mobile terminal $i$. Furthermore, $\mathbf{x}^{d l}=$ $\left[x_{1}^{d l}, \cdots, x_{N}^{d l}\right]^{\mathrm{T}}$ with $x_{i}^{d l}(i=1, \cdots, N)$ being a signal transmitted to mobile terminal $i . \mathbf{W}^{d l}$ is a spatial pre-filter applied at the base station with the constraint

$$
\left\|\mathbf{W}^{d l}\right\|^{2}=N
$$

where $\|$.$\| is the Frobenius norm.$ 
After accounting for proper vector and matrix dimensions and changing the superscripts ' $u l$ ' to ' $d l$ ', the definitions given in the equations (1) to (4) are directly applicable to the downlink. As in the case of the uplink, unique orthogonal pilot waveforms are assigned to each mobile terminal, while the data-carrying waveforms are reused among the mobile terminals. Note that both the pilot and data-carrying portion of the transmitted signal is filtered by the spatial pre-filter $\mathbf{W}^{d l}$.

The application of the spatial pre-filtering results in a composite downlink channel matrix $\mathbf{G}^{d l}$ given as

$$
\mathbf{G}^{d l}=\mathbf{H}^{d l} \mathbf{W}^{d l}, \quad \mathbf{G}^{d l} \in \mathcal{C}^{N \times N} .
$$

Using the pilot assisted estimation, the maximum likelihood estimate of the composite downlink channel matrix $\mathbf{G}^{d l}$ is

$$
\widehat{\mathbf{G}}^{d l}=\mathbf{G}^{d l}+\mathbf{G}_{e}^{d l}
$$

where $\mathbf{G}_{e}^{d l}$ is the estimation error matrix whose entries are modeled as independent identically distributed random variables with complex Gaussian distribution $\mathcal{N}_{\mathcal{C}}\left(0, N_{0} / P_{p}^{d l}\right)$. The decision statistics corresponding to the downlink of mobile terminal $i$ is

$\hat{y}_{i k}^{d l}=\sqrt{P_{d}^{d l}} d_{i k}^{d l} \hat{g}_{i i}^{d l}+\underbrace{\sum_{j=1, j \neq i}^{N} \sqrt{P_{d}^{d l}} d_{j k}^{d l} g_{i j}^{d l}}_{\text {Interference }}+\underbrace{n_{i}^{d l}-\sqrt{P_{d}^{d l}} d_{i k}^{d l} g_{\text {eii }}^{d l}}_{\text {Effective noise }}$

where $\hat{g}_{i i}^{d l}$ is the $i$ th diagonal element of the matrix $\widehat{\mathbf{G}}^{d l}, g_{i j}^{d l}$ is the $i$ th row and $j$ th column element of the matrix $\mathbf{G}^{d l}$ and $g_{e i i}^{d l}$ is the $i$ th diagonal element of the matrix $\mathbf{G}_{e}^{d l}$, all for $k=1, \cdots,\left(K^{d l}-N\right)$. Based on the above a lower bound on the downlink data rate for mobile terminal $i$ is,

$$
\begin{aligned}
C_{i}^{d l} & \geq R_{i}^{d l}=\frac{K^{d l}-N}{K^{d l}} \mathrm{E}_{\mathbf{G}^{d l}, \widehat{\mathbf{G}}^{d l}, \mathbf{G}_{e}^{d l}}\left[\log _{2}(1+\right. \\
& \left.\left.+\frac{P_{d}^{d l}\left|\hat{g}_{i i}^{d l}\right|^{2}}{\sum_{j=1, j \neq i}^{N} P_{d}^{d l}\left|g_{i j}^{d l}\right|^{2}+N_{0}+P_{d}^{d l}\left|g_{e i i}^{d l}\right|^{2}}\right)\right] .
\end{aligned}
$$

\section{Spatial Filter}

In the following the base station assumes that $\mathbf{H}^{u l}=\widehat{\mathbf{H}}^{u l}$, i.e., it ignores the fact that $\mathbf{H}^{u l} \neq \widehat{\mathbf{H}}^{u l}$. The linear multiuser detector that is applied as the uplink receiver is a spatial filter

$$
\mathbf{W}^{u l}=\left(\left(\widehat{\mathbf{H}}^{u l}\right)^{\mathrm{H}} \widehat{\mathbf{H}}^{u l}+\frac{N_{0}}{P_{d}^{u l}} \mathbf{I}\right)^{-1}\left(\widehat{\mathbf{H}}^{u l}\right)^{\mathrm{H}} .
$$

Note that in the case of $\mathbf{H}^{u l}=\widehat{\mathbf{H}}^{u l}$, the above spatial filter minimizes the mean square error, i.e., it is the linear MMSE multiuser detector. It is well known that the MMSE receiver is the optimal linear receiver that maximizes the received SINR (and rate) for each user on the uplink [9].

Due to the reciprocity between the uplink and downlink channels, the downlink transmitter assumes that $\mathbf{H}^{d l}=$ $\left(\mathbf{H}^{u l}\right)^{\mathrm{T}}$ and that their estimates are $\widehat{\mathbf{H}}^{d l}=\left(\widehat{\mathbf{H}}^{u l}\right)^{\mathrm{T}}$. The transmitter optimization that is applied in the downlink transmitter is a spatial pre-filter

$$
\begin{gathered}
\mathbf{W}^{d l}=\mathbf{U P}=\left(\widehat{\mathbf{H}}^{d l}\right)^{\mathrm{H}}\left(\widehat{\mathbf{H}}^{d l}\left(\widehat{\mathbf{H}}^{d l}\right)^{\mathrm{H}}+\frac{N_{0}}{P_{d}^{d l}} \mathbf{I}\right)^{-1} \mathbf{P}, \\
\text { for } \quad \widehat{\mathbf{H}}^{d l}=\left(\widehat{\mathbf{H}}^{u l}\right)^{\mathrm{T}} .
\end{gathered}
$$

In the above, the matrix $\mathbf{P}$ is a diagonal matrix such that the constraint in (11) is satisfied. Specifically, the $i$ th diagonal element of $\mathbf{P}$ is selected as

$$
p_{i i}=\sqrt{\frac{\gamma_{i}}{\mathbf{u}_{i}^{\mathrm{H}} \mathbf{u}_{i}}}=\frac{\sqrt{\gamma_{i}}}{\left|\mathbf{u}_{i}\right|}
$$

where $\mathbf{u}_{i}$ is the $i$ th column vector of the matrix $\mathbf{U}$ in (17), for $i=1, \cdots, N$.

Let us consider an idealized case where (i) the uplink and downlink channels are identical (i.e., $\mathbf{H}^{d l}=\left(\mathbf{H}^{u l}\right)^{\mathrm{T}}$ ), (ii) the channel states are perfectly known (i.e., $\mathbf{H}^{u l}=\widehat{\mathbf{H}}^{u l}$ and $g_{i i}^{d l}=\hat{g}_{i i}^{d l}$ ), (iii) the transmit powers on the uplink and downlink are identical (i.e., $P_{d}^{u l}=P_{d}^{d l}$ ) as well as (iv) the transmission periods (i.e., $K^{u l}=K^{d l}$ ). For the above idealizations, the quantity $\gamma_{i}$ in (18) can be determined such that the multiuser detector in (16) and transmitter optimization in (17) achieve identical uplink and downlink rates, $R_{i}^{u l}=$ $R_{i}^{d l}$, for $i=1, \cdots, N$. The details are given in Appendix.

\section{NUMERICAL RESUlts}

In the following numerical results the channel ${ }^{1}$ between each base station and mobile terminal antenna is modeled as a complex Gaussian unit-variance random variable with the zero mean. Furthermore, the channels are constant for the duration of uplink and downlink transmission period. In other words, we assume a block fading model where each block lasts $K^{u l}+K^{d l}$ symbols, where the channel coherence time is equal or shorter than the block duration. The channel states are independent between different blocks. The uplink and downlink transmission periods are equal, $K=K^{u l}=K^{d l}$.

In Figure 1 we present the downlink rates in (15) as a function of the power allocated to the pilots (percentage wise). The number of the base station antennas is $M=4$ and the mobile terminals is $N=4$. The results are presented for different values of $P^{u l} / N_{0}=P^{d l} / N_{0}$ and the uplink and downlink transmission periods. From the results, we note that the rates have a broad maximum, and the system is not sensitive to exact selection of the pilot power. For example, selecting the pilot power to be $20 \%$ of the total power will accommodate a range of the signal-to-noise power ratios as well as the transmission periods.

In Figure 2 we present the downlink rates as a function of the signal-to-noise power ratio $S N R .20 \%$ of the total power is allocated to the pilots. In this particular example, for the shortest transmission periods $\left(K^{u l}=K^{d l}=25\right)$ the loss

${ }^{1}$ Note that this may correspond to a case when a power control mechanism eliminates effects of large-scale fading (i.e., path loss and shadowing) allowing statistically identical and independent small-scale fading between the base station and mobile terminals (which is captured by the Rayleigh channel model). 


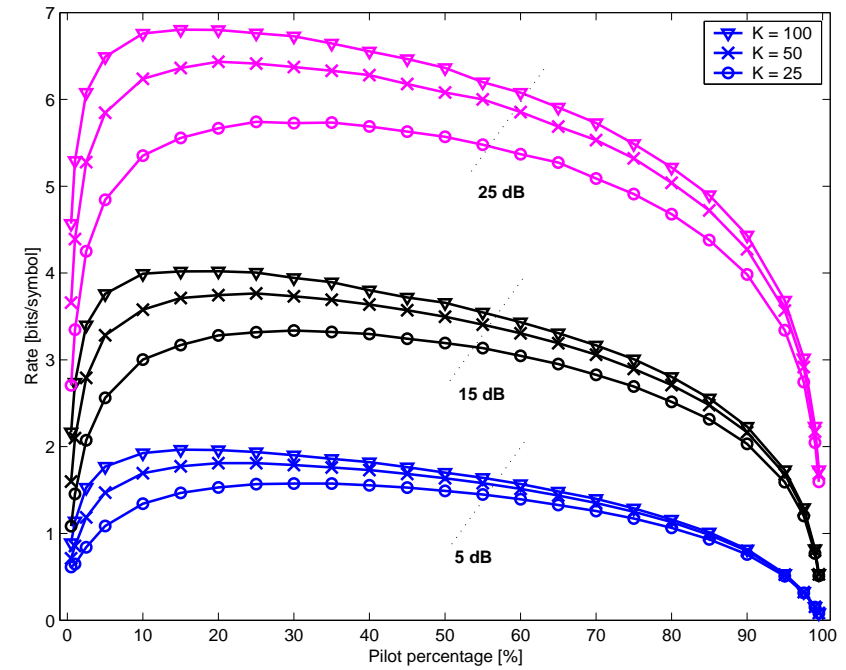

Fig. 1. The downlink rates versus the power allocated to the pilots, for $K=$ $25,50,100$ and $S N R=10 \log \left(P^{u l} / N_{0}\right)=10 \log \left(P^{d l} / N_{0}\right)=5,15,25$ dB.

compared to the idealized case exceeds $3 \mathrm{~dB}$. This may point to a need for a non-coherent scheme, instead of a coherent one.

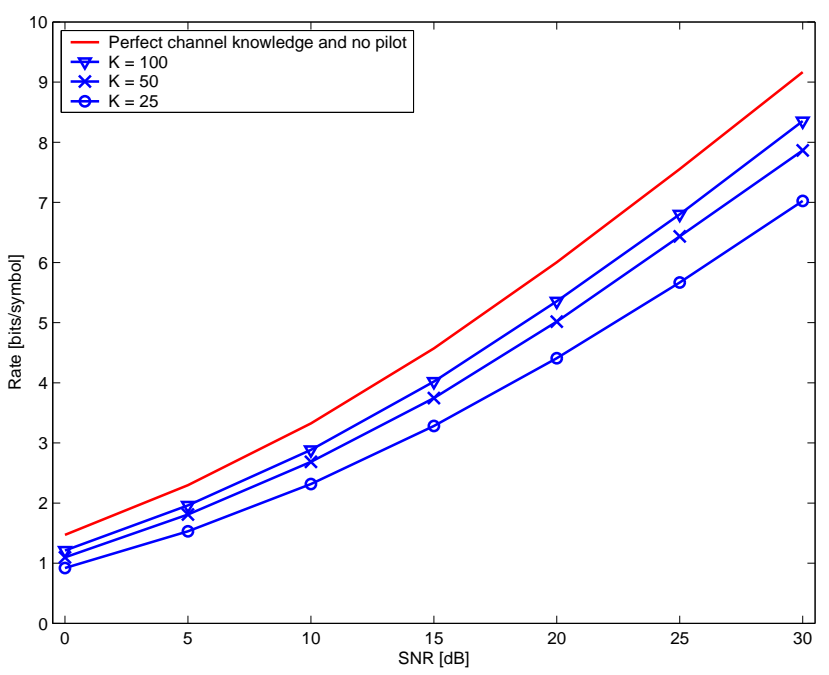

Fig. 2. The downlink rates versus $S N R, \mu^{u l}=\mu^{d l}=20 \%$.

In Figure 3 we present the downlink rates as a function of the number of base station antennas $M$ for $N=4$. The rates increase with the number of antennas following the idealized case. In Figures 1 to 3, the corresponding uplink rates in (9) are more or less identical to that of the presented downlink rates.

Let us now address temporal variations between successive uplink and downlink channel states. It is described using the correlation

$$
k=\mathrm{E}\left[h_{m n}^{u l}\left(h_{n m}^{d l}\right)^{*}\right]
$$

for $n=1, \cdots, N$ and $m=1, \cdots, M$. Low values of the correlation $k$ correspond to higher temporal variations, i.e.,

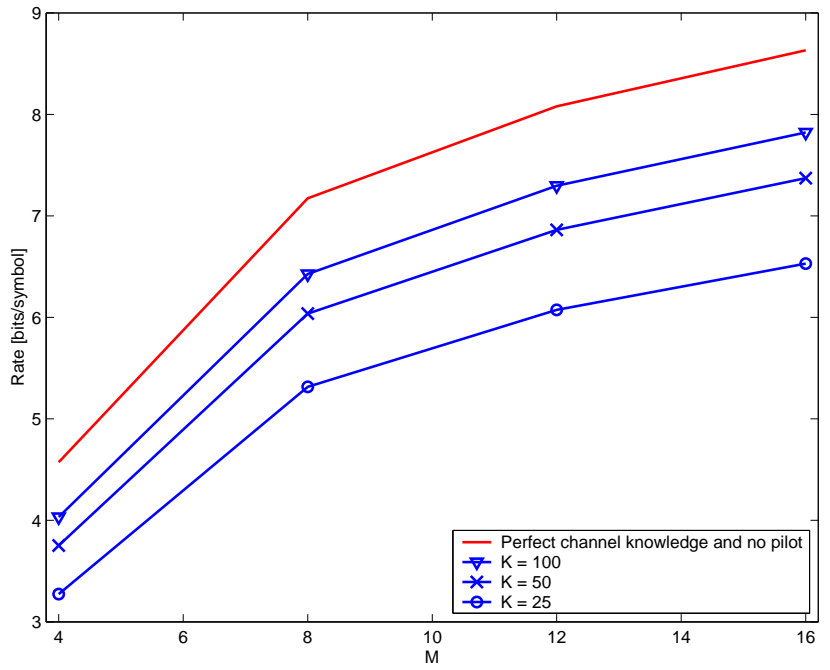

Fig. 3. The downlink rates versus the number of base station antennas $M$, $S N R=15 \mathrm{~dB}, \mu^{u l}=\mu^{d l}=20 \%, K=100$.

mismatch between $h_{m n}^{u l}$ and $h_{n m}^{d l}$. In Figure 4 we present the rates as a function of the correlation $k$. Because of the assumed model, the uplink rates are only affected by the estimation errors. However, the downlink rates are affected by the estimation errors and the correlation $k$. From these results we note that for very rapidly changing channels (low values of $k$ ), the downlink rates are affected quite dramatically. This calls for more frequent channel updates which could be accomplished by using shorter block lengths for transmission. An interesting aspect of future study in this context involves identifying the tradeoff in regards to increased pilot power allocation.

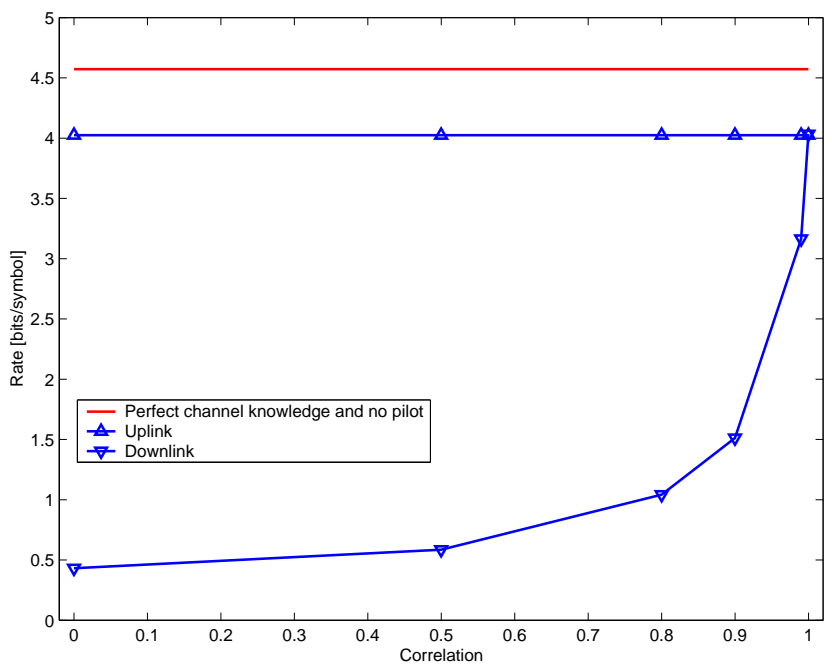

Fig. 4. The rates versus the correlation between successive uplink and downlink channel states, $S N R=15 \mathrm{~dB}, \mu^{u l}=\mu^{d l}=20 \%, K=100$.

\section{Conclusions}

In this paper we have presented a multiple antenna multiuser TDD system that uses pilot assisted channel state estimation. 
We have analyzed the performance of the uplink multiuser detection and downlink transmitter optimization that are based on linear spatial filtering. A lower bound on the achievable rates is presented. Using a block fading channel model we have studied how the lower bound depends on the power allocated to the pilots as well as the channel coherence time. We have shown that the system performance is relatively insensitive to a wide range of the percentage ( $10 \%$ to $40 \%$ ) of the total power allocated to the pilot. In addition, we have demonstrated that the downlink performance is very sensitive to channel variations that call for more frequent channel updates.

\section{ACKNOWLEDGMENT}

This work is supported in part by the NSF under Grant No. 0429724 .

\section{APPENDIX}

In this appendix we present details how to determine the quantities $\gamma_{i}$, for $i=1, \cdots, N$ in (18). For the idealized case of the perfect channel knowledge where $\mathbf{H}^{u l}=\widehat{\mathbf{H}}^{u l}$ the multiuser detector in (16) becomes

$$
\mathbf{W}^{u l}=\left(\left(\mathbf{H}^{u l}\right)^{\mathrm{H}} \mathbf{H}^{u l}+\frac{N_{0}}{P_{d}^{u l}} \mathbf{I}\right)^{-1}\left(\mathbf{H}^{u l}\right)^{\mathrm{H}} .
$$

Likewise, for the static case where $\mathbf{H}^{d l}=\left(\mathbf{H}^{u l}\right)^{\mathrm{T}}$, the transmitter optimization in (17) becomes

$$
\mathbf{W}^{d l}=\left(\mathbf{H}^{d l}\right)^{\mathrm{H}}\left(\mathbf{H}^{d l}\left(\mathbf{H}^{d l}\right)^{\mathrm{H}}+\frac{N_{0}}{P_{d}^{d l}} \mathbf{I}\right)^{-1} \mathbf{P} .
$$

Furthermore, for $P_{d}^{d l}=P_{d}^{u l}$

$$
\mathbf{W}^{d l}=\left(\mathbf{W}^{u l}\right)^{\mathrm{T}} \mathbf{P} .
$$

For the $i$ th mobile terminal, the uplink signal-to-interference and noise power ratio is

$$
S I N R_{i}^{u l}=\frac{P_{d}^{u l}\left|\overline{\mathbf{w}}_{i}^{u l} \mathbf{h}_{i}^{u l}\right|^{2}}{\sum_{j=1, j \neq i}^{N} P_{d}^{u l}\left|\overline{\mathbf{w}}_{i}^{u l} \mathbf{h}_{j}^{u l}\right|^{2}+N_{0}\left|\overline{\mathbf{w}}_{i}^{u l}\right|^{2}}
$$

where $\overline{\mathbf{w}}_{i}^{u l}$ is the $i$ th row vector of the matrix $\mathbf{W}^{u l}$ while $\mathbf{h}_{i}^{u l}$ is the $i$ th column and $\mathbf{h}_{j}^{u l}$ is the $j$ th column vector of the matrix $\mathbf{H}^{u l}$.

For the spatial pre-filtering in (21), the corresponding downlink SINR is

$$
S I N R_{i}^{d l}=\frac{P_{d}^{d l}\left|\overline{\mathbf{h}}_{i}^{d l} \mathbf{w}_{i}^{d l}\right|^{2}}{\sum_{j=1, j \neq i}^{N} P_{d}^{d l}\left|\overline{\mathbf{h}}_{i}^{d l} \mathbf{w}_{j}^{d l}\right|^{2}+N_{0}}
$$

where $\overline{\mathbf{h}}_{i}^{d l}$ is the $i$ th row vector the matrix $\mathbf{H}^{d l} \cdot \mathbf{w}_{i}^{d l}$ is the $i$ th column and $\mathbf{w}_{j}^{d l}$ is the $j$ th column vector of the matrix $\mathbf{W}^{d l}$. Based on the assumptions that led to the result in (22) we rewrite the above $S I N R_{i}^{d l}$ as

$$
S I N R_{i}^{d l}=\frac{\gamma_{i} P_{d}^{u l}\left|\overline{\mathbf{w}}_{i}^{u l} \mathbf{h}_{i}^{u l}\right|^{2} /\left|\overline{\mathbf{w}}_{i}^{u l}\right|^{2}}{\sum_{j=1, j \neq i}^{N} \gamma_{j} P_{d}^{u l}\left|\overline{\mathbf{w}}_{j}^{u l} \mathbf{h}_{i}^{u l}\right|^{2} /\left|\overline{\mathbf{w}}_{j}^{u l}\right|^{2}+N_{0}} .
$$

As a design criterion our goal is to determine the quantities $\gamma_{i}$ that will result in the identical uplink and downlink rates,
$R_{i}^{u l}=R_{i}^{d l}$, for $i=1, \cdots, N$. We define the following set of equations,

$$
S I N R_{i}^{d l}=S I N R_{i}^{u l}, \text { for } i=1, \cdots, N .
$$

They lead to the identical uplink and downlink rates. In addition, the equations are linear.

Let us now define a matrix $\mathbf{T}$ with the following entries

$$
t_{i i}=\sum_{j=1, j \neq i}^{N} \frac{\left|\overline{\mathbf{w}}_{i}^{u l} \mathbf{h}_{j}^{u l}\right|^{2}}{\left|\overline{\mathbf{w}}_{i}^{u l}\right|^{2}}+\frac{N_{0}}{P_{d}^{u l}}
$$

and

$$
t_{i j}=-\frac{\left|\overline{\mathbf{w}}_{j}^{u l} \mathbf{h}_{i}^{u l}\right|^{2}}{\left|\overline{\mathbf{w}}_{j}^{u l}\right|^{2}}, \quad i \neq j
$$

for $i=1, \cdots, N$ and $j=1, \cdots, N$. Furthermore we define a vector $\mathbf{v}$ with the following entries

$$
v_{i}=\frac{N_{0}}{P_{d}^{u l}} \text { for } i=1, \cdots, N .
$$

Using the above definitions, the system of linear equations in (26) can be expressed as

$$
\mathbf{T} \gamma=\mathbf{v}
$$

where $\gamma=\left[\gamma_{1} \cdots \gamma_{N}\right]^{\mathrm{T}}$. Thus, the solution is

$$
\gamma=\left[\gamma_{1} \cdots \gamma_{N}\right]^{\mathrm{T}}=\mathbf{T}^{-1} \mathbf{v} .
$$

Furthermore, based on the equations in (26) it can be shown that

$$
\gamma_{1}+\cdots+\gamma_{N}=N
$$

which satisfies the constraint in (11) with equality. The above result can be obtained by summing column elements of the vectors on both sides of the equation (30).

\section{REFERENCES}

[1] J. H. Winters, J. Salz, and R. D. Gitlin, "The impact of antenna diversity on the capacity of wireless communication systems," IEEE Transactions on Communications, vol. 42, pp. 1740-1751, February/March/April 1994.

[2] G. Caire and S. Shamai, "On the achievable throughput of a multiantenna Gaussian broadcast channel," IEEE Transactions on Information Theory, vol. 49, pp. 1691-1706, July 2003.

[3] E. Rashid-Farrohi, L. Tassiulas, and K. Liu, "Joint optimal power control and beamforming in wireless networks using antenna arrays," IEEE Transactions on Communications, vol. 46, pp. 1313-1323, October 1998.

[4] E. Visotsky and U. Madhow, "Optimum beamforming using transmit antenna arrays," IEEE Vehicular Technology Conference (VTC), vol. 1, pp. 851-856, May 1999.

[5] D. Samardzija, N. Mandayam, and D. Chizhik, "Adaptive transmitter optimization in multiuser multiantenna systems: theoretical limits, effect of delays and performance enhancements," EURASIP Journal on Wireless Communications and Networking, pp. 298-307, August 2005.

[6] D. Samardzija and N. Mandayam, "Unquantized and uncoded channel state information feedback on wireless channels," IEEE Wireless Communications and Networking Conference (WCNC), pp. 1059-1065, March 2005.

[7] R. G. Gallager, Information Theory and Reliable Communications. New York: John Wiley and Sons, 1968.

[8] D. Samardzija and N. Mandayam, "Pilot assisted estimation of MIMO fading channel response and achievable data rates," IEEE Transactions on Signal Processing, Special Issue on MIMO, vol. 51, pp. 2882-2890, November 2003.

[9] U. Madhow and M. Honig, "MMSE interference suppression for directsequence spread-spectrum CDMA," IEEE Transactions on Communications, vol. 42, pp. 3178-3188, December 1994. 